\title{
乌s \\ Partial derivative games in thermodynamics: A cognitive task analysis
}

\author{
Mary Bridget Kustusch* \\ Department of Physics, DePaul University, 2219 North Kenmore Avenue Suite 211, Chicago, Illinois 60614, USA \\ David Roundy, Tevian Dray, and Corinne A. Manogue \\ Department of Physics, Oregon State University, 301 Weniger Hall, Corvallis, Oregon 97331, USA ${ }^{\dagger}$
}

(Received 16 August 2013; published 10 January 2014)

\begin{abstract}
Several studies in recent years have demonstrated that upper-division students struggle with the mathematics of thermodynamics. This paper presents a task analysis based on several expert attempts to solve a challenging mathematics problem in thermodynamics. The purpose of this paper is twofold. First, we highlight the importance of cognitive task analysis for understanding expert performance and show how the epistemic games framework can be used as a tool for this type of analysis, with thermodynamics as an example. Second, through this analysis, we identify several issues related to thermodynamics that are relevant to future research into student understanding and learning of the mathematics of thermodynamics.
\end{abstract}

DOI: 10.1103/PhysRevSTPER.10.010101

PACS numbers: 01.40.Fk, 05.70.-a

\section{INTRODUCTION}

In a recent review of problem solving in physics, Maloney identified several open questions in the area of problem solving, including the following: "How do novices transition to expertise? What are the patterns in how peoples' domain knowledge, problem-solving skills, epistemology, etc., morph from novice status to expert status?" [1]. In order to address these questions, one must first establish what expertise looks like. However, as Maloney points out, a task is not inherently a problem-a problem exists only in the interaction between an individual and a task.

Different people interacting with the same task/situation might not all find it to be a problem. The skills and knowledge an individual brings to a situation play a major role in whether that individual thinks of a situation as a problem. [1]

There have been few studies that have looked at expert problem solving within the domain of physics, and most of those that do, focus on "end-of-chapter" problems in introductory physics which are typically not problems for experts. One of the few exceptions to this is Singh [2], where the given task, although it could be solved using introductory physics concepts and techniques, was nonintuitive and therefore posed a challenge to both physics students and professors.

\footnotetext{
*mkustus1@depaul.edu

${ }^{\dagger}$ http://physics.oregonstate.edu/portfolioswiki/
}

Published by the American Physical Society under the terms of the Creative Commons Attribution 3.0 License. Further distribution of this work must maintain attribution to the author(s) and the published article's title, journal citation, and DOI.
One area of problem solving that is of particular interest to physics faculty is the use of mathematics and the connections between mathematics and physics. In particular, several studies in recent years demonstrate that students struggle to connect mathematics and physics [3-5]. In particular, students struggle with many mathematical aspects of thermodynamics [6-8] and with partial derivatives in particular [9-12]. With few exceptions [13], research on student understanding of derivatives focuses on conceptual understanding of ordinary derivatives, particularly on rate of change and graphical understanding [14-17].

As a part of one author's (D. R.) recent work in redesigning the junior-level thermodynamics course at Oregon State University [18], three authors (D. R., C. A. M., and T. D.). began to explore the uses of differentials within the context of thermodynamics. This was a logical extension of the Vector Calculus Bridge Project [19], which found the vector differential to be a helpful bridge between vector calculus and upper-division electromagnetism courses and led to substantial reforms in how these courses are taught at Oregon State University [20,21].

The current study grew out of these discussions and is part of a larger project designed to better understand how and why students struggle with the mathematics of thermodynamics, how practicing physicists approach the connections between mathematics and physics in thermodynamics, and ultimately how to facilitate an appropriate transition from student to professional in this area.

The purpose of this paper is twofold. First, we highlight the importance of cognitive task analysis for understanding expert performance, particularly for tasks that can be validly addressed in different ways, and we use the epistemic games framework as a tool for this analysis. Second, through this analysis, we identify several issues related to expert use of partial derivatives in thermodynamics that are 
relevant to future research into student understanding and learning of the mathematics of thermodynamics.

In Sec. II, we present the analytical frameworks we are using and follow that with a brief discussion of the study design in Sec. III. We then present descriptions of three epistemic games (Sec. IV) and two variations (Sec. V) that were observed in this study. Section VI compares the games presented here to each other and to previous games identified in the literature, as well as discusses some of the interesting questions raised by this study. Finally, we present some implications and limitations of this work and discuss the directions this work will go in the future (Sec. VII).

\section{BACKGROUND}

This section presents an overview of cognitive task analysis and epistemic games as the primary theoretical frameworks used in this study.

\section{A. Cognitive task analysis}

Research on expertise has a long history and a rich array of methodologies available for analyzing expert performance, including task analysis, which can be succinctly described as "the study of how work is achieved by tasks" [22]. In The Cambridge Handbook of Expertise and Expert Performance [23], Schraagen provides an excellent overview of the historical progression of task analysis and an introduction to some of the approaches within this field that focus on professional practitioners (e.g., hierarchical task analysis, critical incident technique, etc.) [24].

Given the cognitive nature and importance of disciplinary knowledge for many of the tasks in physics, the emergence of cognitive task analysis, primarily within the human-computer interaction community, is of particular relevance to the physics education research community.

Cognitive task analysis is the extension of traditional task analysis techniques to yield information about the knowledge, thought processes, and goal structures that underlie observable task performance. [25]

There are many different methods that fall under the umbrella of cognitive task analysis, and they typically focus on tasks that are highly discipline specific and complex, something that is vital for understanding expertise in our context.

Task analysis now has a focus of understanding expert knowledge, reasoning, and performance, and leveraging that understanding into methods for training and decision support, to amplify and extend human abilities to know, perceive, and collaborate. [24]

This paper presents a cognitive task analysis for a mathematical task in thermodynamics at the upperdivision undergraduate level. At this level, students have already transitioned from novices to something more akin to journeymen [26]. Even well-defined "end-of-chapter" problems have become more complex and there are often multiple ways to solve the same problem. To understand and describe problem solving that is correspondingly more complex and discipline specific requires leveraging the strengths of a field of inquiry that is as rich and diverse as cognitive task analysis.

\section{B. Epistemic games}

As mentioned above, there are many different methods for performing a cognitive task analysis. One that we have found particularly fruitful in this case is the framework of epistemic games, originally proposed by Collins and Ferguson [27], who define an epistemic game as a complex "set of rules and strategies that guide inquiry." They use these games as a way to describe expert scientific behavior that could be considered normative within a given community. The use of epistemic games has since been extended in several ways, primarily as a descriptive analysis tool for understanding student behavior and classroom discourse [28-34].

This paper proposes three new epistemic games and two variations as a means of providing a task analysis for a challenging thermodynamics problem. We extract the commonalities in the behavior of experts as a means of understanding ways of thinking that are reflective of the community. In this sense, we are consistent with Collins and Ferguson's use of epistemic games as depicting normative practices. However, we also draw these conclusions from the behavior of specific experts, similar to other studies that are more descriptive in nature (e.g., Tuminaro and Redish [34]). Including both descriptive and normative elements allows for a discussion of what we currently and implicitly expect our students to learn and provokes questions such as whether the practices within the community serve the purposes we think they do and, if so, whether the use of certain epistemic games should be more explicit in our instruction.

It is important to note that there are different perspectives on the specificity of epistemic games. Perkins [35] claims that epistemic games are a high-level, decontextualized form of reasoning and some of the epistemic games that have since been proposed are also in this camp (e.g., the answer-making epistemic game proposed by Chen et al. [28]), while others are more discipline specific (e.g., Lunk's [29] iterative-debugging game). Most of the games proposed by Collins and Ferguson are fairly discipline independent (e.g., list game, compareand-contrast game, cause-and-effect game, etc.), but they also point out that

\footnotetext{
Different disciplines are characterized by the forms and games they use. As disciplines evolve, they develop more complex and more constrained epistemic forms and games. These are sometimes specialized to fit the subject matter being analyzed.
} 
Additionally, even Perkins allows that there are both general and specific forms of epistemic games. Given the goals of this project and the subject matter at hand, we believe a discipline-specific perspective is not only appropriate but necessary. Thus, the games proposed in this paper are highly specialized and constrained.

Here we introduce the terminology and relevant features of epistemic games used throughout this paper. Collins and Ferguson [27] outline several elements of epistemic games: entry conditions, moves, constraints, transfers, and a target epistemic form. Tuminaro and Redish [34] categorize components of epistemic games according to whether they are ontological or structural (see Table I). Unlike Collins and Ferguson, they give explicit attention to the "cognitive resources associated with the game," which they call the "knowledge base." Tuminaro and Redish also appear to group moves, constraints, and transfers into one component, which they call "moves."

The most important element of an epistemic game for Collins and Ferguson is the epistemic form, which they define as "the target structures that guide scientific inquiry" and they identify different games primarily based on their target epistemic form. They use an analogy to tictac-toe to distinguish between the epistemic game and the epistemic form, where "the difference between forms and games is like the difference between the squares that are filled out in tic-tac-toe and the game itself."

Collins and Ferguson introduce three broad categories of epistemic games-structural analysis games, functional analysis games, and process analysis games-and discuss several example games in each category. The canonical example that they use throughout the paper is the list game. This falls under the category of structural analysis games, where the primary goal is to determine the nature of some phenomenon "by breaking [it] down into subsets or constituents and describing the relationships among the constituents." Most of the games in this category focus on characterization and/or categorization.

In contrast, each of the epistemic games we present here belongs to the category of functional analysis games, [36] where "the goal is to determine the causal or functional

TABLE I. The ontological and structural components of epistemic games. (Reproduced from Tuminaro and Redish [34].)

\begin{tabular}{lc}
\hline \hline Knowledge base & $\begin{array}{c}\text { Ontological components } \\
\text { with the game } \\
\text { Target structure that guides inquiry }\end{array}$ \\
\hline $\begin{array}{l}\text { Entry and ending } \\
\text { conditions } \\
\text { Moves }\end{array}$ & $\begin{array}{c}\text { Conditions for when to begin and end } \\
\text { playing a particular game } \\
\text { Activities that occur during the course } \\
\text { of an e-game }\end{array}$ \\
\hline \hline
\end{tabular}

structures that relate elements in a system" [27]. In the context of the current study, this goal primarily manifests as an attempt to answer the following question: Which quantities depend on which other quantities; i.e., which quantities are constant, which are independent variables, which are dependent variables, etc.?

In most physics subdisciplines, this functional structure is known and taking a partial derivative is a matter of simply playing the controlling-variables game,

in which one tries to manipulate one variable at a time while holding other variables constant in order to determine the effect of each independent variable on the dependent variable. [27]

However, one of the challenges of thermodynamics is that the relationships between quantities are not always readily apparent and thus dealing with partial derivatives involves first playing a functional analysis game.

All of the games we discuss here attempt to answer the same question about the functional structure of a problem, but in fundamentally different ways. Although the games share some moves and there is a large overlap in their requisite knowledge base, they differ significantly in their target epistemic form and in a few key moves. Our discussion primarily focuses on these differences since they raise some interesting questions that are relevant to thermodynamics instruction.

\section{STUDY DESIGN}

As mentioned earlier, this work arose from on-going discussions among three of the authors (C. A. M., T.D., D.R.) regarding the possibility of using differentials as a bridge for understanding partial derivatives within thermodynamics. The first author (M. B. K.) had not been a part of the earlier conversations and curriculum development. She conducted interviews with each of the three coauthors (who will continue to be referred to by their initials) and seven additional faculty members from various institutions who have experience teaching thermodynamics (referred to by pseudonyms). Table II describes the background of each of these experts. Each participant (except Sam [37]) was asked to think aloud as they worked through the following problem:

Find $\left(\frac{\partial U}{\partial p}\right)_{S}$ for a van der Waals gas, given the following equations of state:

$$
\begin{gathered}
p=\frac{N k T}{V-N b}-\frac{a N^{2}}{V^{2}}, \\
S=N k\left\{\ln \left[\frac{(V-N b) T^{3 / 2}}{N \Phi}\right]+\frac{5}{2}\right\},
\end{gathered}
$$


TABLE II. Background of faculty interviewees, including relevant teaching experience and education research. Some also conduct traditional physics research involving thermodynamics, which we have not included. Although we only mention thermodynamics below, many of the courses include a significant statistical mechanics portion as well.

\begin{tabular}{|c|c|c|c|}
\hline Name & Institution & Discipline & Relevant education research or teaching experience \\
\hline Leo & Undergraduate & Physics & Teaches upper-level undergraduate thermodynamics \\
\hline Chris & $\begin{array}{l}\text { (Nontraditional) } \\
\text { Undergraduate }\end{array}$ & Physics & $\begin{array}{l}\text { Teaches upper-level undergraduate thermodynamics in an interdisciplinary } \\
\text { and nontraditional sequence }\end{array}$ \\
\hline Sam & Undergraduate & Physics & Physics education research on thermodynamics \\
\hline Elliott & M.S. granting & Physics & $\begin{array}{l}\text { Physics education research on thermodynamics; teaches upper-level } \\
\text { undergraduate thermodynamics }\end{array}$ \\
\hline Jay & Ph.D. granting & Physics & Teaches upper-level undergraduate and graduate-level thermodynamics \\
\hline Gary & Ph.D. granting & Physics & Teaches upper-level undergraduate and graduate-level thermodynamics \\
\hline Keith & Ph.D. granting & Engineering & $\begin{array}{l}\text { Engineering education research; teaches chemical engineering } \\
\text { thermodynamics }\end{array}$ \\
\hline D. R. & Ph.D. granting & Physics & Coauthor, Energy and Entropy instructor \\
\hline C. A. M. & Ph.D. granting & Physics & Coauthor, director of Paradigms in Physics Project \\
\hline T. D. & Ph.D. granting & Math & Coauthor, director of Vector Calculus Bridge Project \\
\hline
\end{tabular}

$$
U=\frac{3}{2} N k T-\frac{a N^{2}}{V} .
$$

Before proceeding, we suggest that the reader consider how they might approach this problem.

Given the focus of the paper on understanding expert approaches to mathematical problems within thermodynamics, this problem was designed to have clear initial and final states (i.e., the given values and the quantity to be found), but allow for multiple approaches. The goal was to have a problem similar to those that students might encounter in an upper-division thermodynamics course, but which would be mathematically complex enough to be a challenge for the experts. Not counting coauthors, who had helped to design the problem, only Leo and Sam fully solved the problem with no errors, indicating that this task was indeed a problem for these experts.

In order to perform the cognitive task analysis, the first author drew on video, transcripts, and written work from each interview, as well as both formal and informal followup conversations with her three coauthors. Descriptions of each game emerged by analyzing each experts' moves and stated justifications for those moves. For example, if an individual expert distinguished between two approaches, this was an indication that the individual viewed these as distinct activities. Similarly, if two experts used similar language to talk about similar moves, this was taken as an indication that they were engaged in the same game. Follow-up conversations with coauthors were used as additional checks on the validity of these games.

We identified three primary epistemic games and two variations that these experts played or proposed while solving this problem. There were a few approaches used by the experts that are not covered by these games (e.g., work, heat capacities, etc.). However, we have chosen to report here only on games that were played to completion and/or games that were proposed by a majority of the experts in this study.

\section{MATHEMATICS EPISTEMIC GAMES}

As previously mentioned, the van der Waals problem presented above was designed to be a mathematical problem in a thermodynamics context and most of the experts treated it as such. The amount and type of physical reasoning the experts did varied from individual to individual. This sense-making activity will be briefly discussed in Sec. VI and more fully addressed in a future paper.

This section describes the three epistemic gamesSubstitution, Partial Derivatives, and Differentials-that these experts played or proposed while solving the van der Waals problem. The target epistemic forms for these games and some of the key moves are summarized in Table III and are discussed in detail below.

TABLE III. Summary of target epistemic forms and key moves for each epistemic game.

\begin{tabular}{lcl}
\hline \hline Game & Epistemic form & Key moves and constraints \\
\hline Substitution & $U=U(p, S)$ & $\begin{array}{c}\text { Isolate one variable as function of desired variables; substitute } \\
\text { one expression into another }\end{array}$ \\
Partial derivatives & $\left(\frac{\partial U}{\partial p}\right)_{S}=\left(\frac{\partial \square}{\partial \square}\right)_{\square}\left(\frac{\partial \square}{\partial \square}\right)_{\square}+\cdots$ & $\begin{array}{c}\text { Recursive use of partial derivative rules (Table IV); constraint: } \\
\text { a move must produce nice sets }\end{array}$ \\
Differentials & $d U=\left(\frac{\partial U}{\partial p}\right)_{S} d p+\left(\frac{\partial U}{\partial S}\right)_{p} d S$ & Finding differentials, linear algebra \\
\hline \hline
\end{tabular}




\section{A. Substitution}

The primary goal of the Substitution game is to use a set of functional relationships between certain quantities to discover the relationship between a different set of quantities. Typical moves within this game are to solve an expression for one variable in terms of another variable and to substitute one expression into another. Within the Substitution game, different constraints allow for different sets of moves. For example, if the given relationships are a set of linear equations, one can employ moves from linear algebra that are not appropriate for a set of nonlinear equations.

One instantiation of the goal of the Substitution game for the van der Waals problem would be to take the given equations of state [Eqs. (1)-(3)] that describe internal energy, pressure, and entropy as functions of volume and temperature and find an explicit expression for internal energy as a function of pressure and entropy. This expression for the internal energy is the target epistemic form for this game and is represented in Table III as $U=U(p, S)$. One possible set of moves to achieve this goal within this game is as follows:

(1) Solve Eq. (2), where $S=S(V, T)$, for $V(T, S)$.

(2) Substitute $V(T, S)$ into Eq. (1), where $p=p(T, V)$, to get $p(T, S)$.

(3) Substitute $V(T, S)$ into Eq. (3), where $U=U(T, V)$, to get $U(T, S)$.

(4) Solve $p(T, S)$ for $T(p, S)$.

(5) Substitute $T(p, S)$ into $U(T, S)$, to get $U(p, S)$.

Once the internal energy is explicitly expressed as a function of pressure and entropy, one can take the desired partial derivative directly.

The van der Waals question had been deliberately chosen to make the Substitution game unattractively difficult. Indeed, this game was never, on its own, actually played to completion by any of these experts. This was usually because of a clearly expressed distaste for the algebra involved. For example, Elliott described this game and his decision not to play it when he stated,

So you've given me an analytical expression for $S$, so in principle I could plug that in, you know solve for something and plug that in there [Eq. (3)] and then I have an explicit $S$ dependence, I would hold that [S] constant and take that derivative $\left[(\partial U / \partial p)_{S}\right]$, but that seems like a real pain. So, I'm gonna put that aside for the moment, just because, I don't wanna do that right now.

Although none of these experts played this game to completion, we include it here because all used the Substitution game as a subgame within another gamethis is what Collins and Ferguson [27] refer to as a "transfer": a move that involves transferring to a different game or subgame. For all of the experts in this study, the goals and moves of the Substitution game were used to achieve a subgoal within another game. It is important to note that, in our preliminary analysis of student data, this is the only game that some students consider.

\section{B. Partial Derivatives}

For the van der Waals problem, the Partial Derivatives game was the most common game played among this group of experts. Of the non-coauthor experts, all but Gary played this game to some extent.

The target epistemic form for this game is an expression that relates the desired partial derivative, $(\partial U / \partial p)_{S}$, to partial derivatives that can be directly calculated from given information, which Jay called "nice sets," i.e.,

$$
\begin{array}{ll}
\left(\frac{\partial p}{\partial V}\right)_{T}, & \left(\frac{\partial p}{\partial T}\right)_{V}, \\
\left(\frac{\partial S}{\partial V}\right)_{T}, & \left(\frac{\partial S}{\partial T}\right)_{V}, \\
\left(\frac{\partial U}{\partial V}\right)_{T}, & \left(\frac{\partial U}{\partial T}\right)_{V} .
\end{array}
$$

This target form is expressed in Table III as

$$
\left(\frac{\partial U}{\partial p}\right)_{S}=\left(\frac{\partial \square}{\partial \square}\right)_{\square}\left(\frac{\partial \square}{\partial \square}\right)_{\square}+\cdots,
$$

where the right-hand side of this equation is designed to represent the combination of derivatives from Eq. (4) for which one is looking. Keith summarized this game by stating,

So I wanna use, be able to use these [derivatives in Eq. (4)] to figure out mathematically how to relate $U$ and $p$ at constant $S$.

Thus, unlike the Substitution game, the Partial Derivatives game bypasses the need to find an explicit expression for $U$.

The key moves of this game are the various partial derivative rules shown in Table IV. There is one primary constraint on these moves: whether a move, or combination of moves, yields one or more "nice sets" [derivatives from Eq. (4)]. Every expert who played this game evaluated each step to determine which derivatives could be calculated directly from the equations of state and which still needed to be rewritten in terms of those derivatives. In a previous paper [38], we provided a detailed description of Jay's path through this problem while playing this game. An idealized example of how one might play this game to solve the given problem is provided in Table $\mathrm{V}$.

In order to completely answer the question, there is one more necessary move: to evaluate the derivatives in Eq. (4) from the given equations of state [Eqs. (1)-(3)]. This move can be played at any point in the game, but some experts did not use this move at all, stating as Jay did 
TABLE IV. Several common partial derivative rules.

\begin{tabular}{ll}
\hline \hline Inversion & $\left(\frac{\partial A}{\partial B}\right)_{C}=\left(\frac{\partial B}{\partial A}\right)_{C}^{-1}$ \\
Cyclic chain rule & $\left(\frac{\partial A}{\partial B}\right)_{C}=-\left(\frac{\partial A}{\partial C}\right)_{B}\left(\frac{\partial C}{\partial B}\right)_{A}$ \\
One-dimensional chain rule [for $A=A(C, D)$ and $D=D(B, C)]$ & $\left(\frac{\partial A}{\partial B}\right)_{C}=\left(\frac{\partial A}{\partial D}\right)_{C}\left(\frac{\partial D}{\partial B}\right)_{C}$ \\
Two-dimensional chain rule [for $A=A(D, E)$ and both $D=D(B, C)$ and $E=D(B, C)]$ & $\left(\frac{\partial A}{\partial B}\right)_{C}=\left(\frac{\partial A}{\partial D}\right)_{E}\left(\frac{\partial D}{\partial B}\right)_{C}+\left(\frac{\partial A}{\partial E}\right)_{D}\left(\frac{\partial E}{\partial B}\right)_{C}$ \\
Two-dimensional chain rule (variant) $[$ for $A=A(B, D)$ and $D=D(B, C)]$ & $\left(\frac{\partial A}{\partial B}\right)_{C}=\left(\frac{\partial A}{\partial B}\right)_{D}+\left(\frac{\partial A}{\partial D}\right)_{B}\left(\frac{\partial D}{\partial B}\right)_{C}$ \\
\hline \hline
\end{tabular}

TABLE V. Idealized example of the Partial Derivatives game. Bolded partial derivatives are not "nice sets."

1. Use $2 \mathrm{D}$ chain rule on the energy equation of state [Eq. (3)], where $U=U(V, T)$

2. Rewrite the $(\partial T / \partial p)_{S}$ term

$$
\left(\frac{\partial U}{\partial p}\right)_{S}=\left(\frac{\partial U}{\partial V}\right)_{T}\left(\frac{\partial V}{\partial p}\right)_{S}+\left(\frac{\partial U}{\partial T}\right)_{V}\left(\frac{\partial T}{\partial p}\right)_{S}
$$

(a) Use $2 \mathrm{D}$ chain rule variant on the $(\partial T / \partial p)_{S}$ term

$$
\left(\frac{\partial T}{\partial p}\right)_{S}=\left(\frac{\partial T}{\partial p}\right)_{V}+\left(\frac{\partial T}{\partial V}\right)_{p}\left(\frac{\partial V}{\partial p}\right)_{S}
$$

(b) Use the cyclic chain rule on the $(\partial T / \partial V)_{p}$ term and invert relevant terms

$$
\begin{gathered}
\left(\frac{\partial T}{\partial V}\right)_{p}=-\left(\frac{\partial T}{\partial p}\right)_{V}\left(\frac{\partial p}{\partial V}\right)_{T} \\
\Rightarrow\left(\frac{\partial T}{\partial p}\right)_{S}=\left(\frac{\partial p}{\partial T}\right)_{V}^{-1}-\left(\frac{\partial V}{\partial p}\right)_{S}\left(\frac{\partial p}{\partial T}\right)_{V}^{-1}\left(\frac{\partial p}{\partial V}\right)_{T}
\end{gathered}
$$

3. Insert new expression for $(\partial T / \partial p)_{S}$ into step 1, rearrange to factor out the $(\partial V / \partial p)_{S}$ term and invert that term

$$
\begin{aligned}
\left(\frac{\partial U}{\partial p}\right)_{S} & =\left(\frac{\partial U}{\partial V}\right)_{T}\left(\frac{\partial V}{\partial p}\right)_{S}+\left(\frac{\partial U}{\partial T}\right)_{V}\left[\left(\frac{\partial p}{\partial T}\right)_{V}^{-1}-\left(\frac{\partial V}{\partial p}\right)_{S}\left(\frac{\partial p}{\partial T}\right)_{V}^{-1}\left(\frac{\partial p}{\partial V}\right)_{T}\right] \\
& =\left(\frac{\partial U}{\partial T}\right)_{V}\left(\frac{\partial p}{\partial T}\right)_{V}^{-1}+\left[\left(\frac{\partial U}{\partial V}\right)_{T}-\left(\frac{\partial U}{\partial T}\right)_{V}\left(\frac{\partial p}{\partial T}\right)_{V}^{-1}\left(\frac{\partial p}{\partial V}\right)_{T}\right]\left(\frac{\partial p}{\partial V}\right)_{S}^{-1}
\end{aligned}
$$

At this point, everything except $(\partial p / \partial V)_{S}$ is a derivative that can be calculated directly from the equations of state

4. Rewrite the $(\partial p / \partial V)_{S}$

(a) Use $2 \mathrm{D}$ chain rule variant on the $(\partial p / \partial V)_{S}$ term

$$
\left(\frac{\partial p}{\partial V}\right)_{S}=\left(\frac{\partial p}{\partial V}\right)_{T}+\left(\frac{\partial p}{\partial T}\right)_{V}\left(\frac{\partial T}{\partial V}\right)_{S}
$$

(b) Use the cyclic chain rule on the $(\partial T / \partial V)_{S}$ term and invert relevant terms

$$
\begin{gathered}
\left(\frac{\partial T}{\partial V}\right)_{S}=-\left(\frac{\partial T}{\partial S}\right)_{V}\left(\frac{\partial S}{\partial V}\right)_{T} \\
\Rightarrow\left(\frac{\partial p}{\partial V}\right)_{S}=\left(\frac{\partial p}{\partial V}\right)_{T}-\left(\frac{\partial p}{\partial T}\right)_{V}\left(\frac{\partial S}{\partial T}\right)_{V}^{-1}\left(\frac{\partial S}{\partial V}\right)_{T}
\end{gathered}
$$

5. Insert new expression for $(\partial p / \partial V)_{S}$ into the expression found in step 3 and simplify

$$
\begin{gathered}
\left(\frac{\partial U}{\partial p}\right)_{S}=\left(\frac{\partial U}{\partial T}\right)_{V}\left(\frac{\partial p}{\partial T}\right)_{V}^{-1}+\left[\left(\frac{\partial U}{\partial V}\right)_{T}-\left(\frac{\partial U}{\partial T}\right)_{V}\left(\frac{\partial p}{\partial T}\right)_{V}^{-1}\left(\frac{\partial p}{\partial V}\right)_{T}\right]\left[\left(\frac{\partial p}{\partial V}\right)_{T}-\left(\frac{\partial p}{\partial T}\right)_{V}\left(\frac{\partial S}{\partial T}\right)_{V}^{-1}\left(\frac{\partial S}{\partial V}\right)_{T}\right]^{-1} \\
\left(\frac{\partial U}{\partial p}\right)_{S}=\frac{\left(\frac{\partial U}{\partial V}\right)_{T}\left(\frac{\partial S}{\partial T}\right)_{V}-\left(\frac{\partial U}{\partial T}\right)_{V}\left(\frac{\partial S}{\partial V}\right)_{T}}{\left(\frac{\partial p}{\partial V}\right)_{T}\left(\frac{\partial S}{\partial T}\right)_{V}-\left(\frac{\partial p}{\partial T}\right)_{V}\left(\frac{\partial S}{\partial V}\right)_{T}}
\end{gathered}
$$


At this point, I have proven, reduced it to all kind of derivatives which I can take from there [points to equations of state, Eqs. (1)-(3)], cause they have the right set, have the right combination of variables in there.

Thus, at that point, he believed, as Keith did, that

I have this thing solved in principle.

Others (e.g., Chris and Sam) only evaluated derivatives after the target form had been obtained. Of those that played the Partial Derivatives game, only Leo made this move early (discussed in more detail in Sec. VA).

\section{Differentials}

The Differentials game was far less common than the Partial Derivatives game - only the three coauthors played this game to completion without prompting. Some experts started this game and then abandoned it (e.g., Keith, Elliott, and Gary) and others played it only after being prompted (e.g., Jay and Sam).

The target epistemic form for this game is

$$
d U=\left(\frac{\partial U}{\partial p}\right)_{S} d p+\left(\frac{\partial U}{\partial S}\right)_{p} d S
$$

where the solution to the van der Waals problem is simply the coefficient of the $d p$ term.

The primary move for this game is to find the differential for each equation of state [Eqs. (1)-(3)]

$$
\begin{gathered}
d p=\left(\frac{\partial p}{\partial T}\right)_{V} d T+\left(\frac{\partial p}{\partial V}\right)_{T} d V \\
d S=\left(\frac{\partial S}{\partial T}\right)_{V} d T+\left(\frac{\partial S}{\partial V}\right)_{T} d V \\
d U=\left(\frac{\partial U}{\partial T}\right)_{V} d T+\left(\frac{\partial U}{\partial V}\right)_{T} d V .
\end{gathered}
$$

One then enters the Substitution game to solve this system of equations to get the target form, where the relevant quantities are now differentials instead of the variables themselves. Since this set of equations is linear in the differentials, one can use either the standard moves of the Substitution game or the moves of linear algebra. Like the Partial Derivatives game, using differentials bypasses the need to find an explicit expression for $U$.

In addition, there is a fundamental difference regarding the moves in the Partial Derivatives game and the primary move in the Differentials game. The constraint on moves in the Partial Derivatives game-whether they yield "nice sets" [Eq. (4)]-is automatically satisfied in the Differentials game. By taking the equations of state and "zapping with $d$ " [21], one is working only with the derivatives in Eq. (4) ("nice sets"). This eliminates the need to evaluate each step for this constraint.

$$
\begin{aligned}
d U & =E d V+F d T \\
d p & =A d V+B d T \\
d s & =C d V+D d T \\
\frac{E D-C F}{A D-B C} & =\frac{\left(\frac{\partial u}{\partial V}\right)_{T}\left(\frac{\partial S}{\partial T}\right)_{V}-\left(\frac{\partial S}{\partial V}\right)_{T}\left(\frac{\partial u}{\partial T}\right)_{T}}{\left(\frac{\partial P}{\partial V}\right)_{T}\left(\frac{\partial S}{\partial T}\right)_{V}-\left(\frac{\partial p}{\partial T}\right)_{V}\left(\frac{\partial S}{\partial V}\right)_{T}}
\end{aligned}
$$

FIG. 1. C. A. M.'s use of letters as placeholders for explicit derivatives and her solution (see Table VI).

Of those that played the Differentials game, some explicitly evaluated the derivatives,

$$
\begin{gathered}
d p=\left[\frac{2 a N^{2}}{V^{3}}-\frac{N k T}{(V-N b)^{2}}\right] d V+\frac{N k}{V-N b} d T, \\
d S=\frac{N k}{V-N b} d V+\frac{3}{2} \frac{N k}{T} d T, \\
d U=\frac{a N^{2}}{V^{2}} d V+\frac{3}{2} N k d T .
\end{gathered}
$$

Others simply used placeholders for those derivatives (see Fig. 1). An idealized example of the Differentials game, based on C.A.M.'s solution and using this kind of notation, is provided in Table VI. This kind of notation is not a useful option in the Partial Derivatives game because the derivatives are constantly being changed around, and it would keep one from recognizing possible moves.

\section{GAME VARIATIONS}

There are two variations that can be played within the games discussed above. These variations can be thought of as possible moves that provide alternate paths through each game. Typically, these alternate paths manifest by providing additional constraints on remaining moves and/or in producing a variant to the epistemic form. This section will briefly describe each variation and how it impacts the different games.

\section{A. Constant entropy as constraint}

The first variation involves explicitly setting entropy equal to a constant and using this constraint to reduce the degrees of freedom for the problem.

In the Differentials game, this move involves setting $d S=0$ explicitly, which both T. D. and Jay did in the differential form of the entropy equation of state [Eq. (2)]:

$$
d S=0=\left(\frac{\partial S}{\partial T}\right)_{V} d T+\left(\frac{\partial S}{\partial V}\right)_{T} d V
$$

This move produces a variation in the epistemic form by eliminating one of the degrees of freedom. Thus, instead of 
TABLE VI. Idealized example of the Differentials game (based on C. A. M.'s solution).

1. Find the differential for each equation of state [Eqs. (1)-(3)], either explicitly evaluating the derivatives or using placeholder names, as below

$$
\begin{aligned}
& d p=\left(\frac{\partial p}{\partial V}\right)_{T} d V+\left(\frac{\partial p}{\partial T}\right)_{V} d T=A d V+B d T \\
& d S=\left(\frac{\partial S}{\partial V}\right)_{T} d V+\left(\frac{\partial S}{\partial T}\right)_{V} d T=C d V+D d T \\
& d U=\left(\frac{\partial U}{\partial V}\right)_{T} d V+\left(\frac{\partial U}{\partial T}\right)_{V} d T=E d V+F d T
\end{aligned}
$$

2. Multiply Eq. (6) by $D$ and Eq. (7) by $-B$, then add to find $d V$ in terms of $d p$ and $d S$

$$
\begin{aligned}
D d p & =+A D d V+B D d T \\
-B d S & =-B C d V-B D d T \\
\Rightarrow D d p-B d S & =(A D-B C) d V \\
d V & =\frac{D d p-B d S}{A D-B C}
\end{aligned}
$$

3. Multiply Eq. (6) by $-C$ and Eq. (7) by $A$, then add to find $d T$ in terms of $d p$ and $d S$

$$
\begin{aligned}
-C d p & =-A C d V-B C d T \\
A d S & =+A C d V+A D d T \\
\Rightarrow-C d p+A d S & =(A D-B C) d T \\
d T & =\frac{-C d p+A d S}{A D-B C}
\end{aligned}
$$

4. Substitute results from steps 2 and 3 into Eq. (8) to get an expression for $d U$ in terms of $d p$ and $d S$

$$
d U=E\left(\frac{D d p-B d S}{A D-B C}\right)+F\left(\frac{-C d p+A d S}{A D-B C}\right)=\left(\frac{E D-F C}{A D-B C}\right) d p+\left(\frac{F A-E B}{A D-B C}\right) d S
$$

5. At this point, the solution is simply the coefficient of the $d p$ term (see Fig. 1), which is equivalent to the solution from the Partial Derivatives game (see Table V)

$$
\left(\frac{\partial U}{\partial p}\right)_{S}=\frac{E D-F C}{A D-B C}=\frac{\left(\frac{\partial U}{\partial V}\right)_{T}\left(\frac{\partial S}{\partial T}\right)_{V}-\left(\frac{\partial U}{\partial T}\right)_{V}\left(\frac{\partial S}{\partial V}\right)_{T}}{\left(\frac{\partial p}{\partial V}\right)_{T}\left(\frac{\partial S}{\partial T}\right)_{V}-\left(\frac{\partial p}{\partial T}\right)_{V}\left(\frac{\partial S}{\partial V}\right)_{T}}
$$

looking for an expression for $d U$ in terms of $d p$ and $d S$, one is looking for an expression for $d U$ in terms of the single variable, $d p$, that is valid only for constant entropy. One can also explicitly hold the entropy constant by setting $d S=0$ in the thermodynamic identity, but since this approach combines two different variations, it will be discussed more fully in Sec. V B.

In the Substitution game, setting entropy equal to a constant can serve as one of several possible starting moves. By explicitly setting the entropy equation of state [Eq. (2)]

$$
S=N k\left\{\ln \left[\frac{(V-N b) T^{3 / 2}}{N \Phi}\right]+\frac{5}{2}\right\}
$$

equal to a constant, one can find an explicit relationship between $T$ and $V$. This relationship can, in turn, be used to find $U$ and $p$ as functions of only one variable, e.g., $U=$ $U(V)$ and $p=p(V)$. As in the Differentials game, solving this new set of equations leads to a variant of the original epistemic form, an expression for internal energy as a function only of pressure, i.e., $U=U(p)$, that is only valid for constant entropy.

In the Partial Derivatives game, one can use this variation by entering the Substitution game as described above to find internal energy and pressure as functions of only one variable. This then places constraints on the remaining moves. Leo, whose written work is shown in Fig. 2, was the only expert to use this move within the Partial Derivatives game. He began by writing

$$
\left(\frac{\partial U}{\partial p}\right)_{S}=\left(\frac{\partial U}{\partial V}\right)_{S}\left(\frac{\partial V}{\partial p}\right)_{S}+\cdots
$$




$$
\begin{aligned}
& P(\psi, T) \\
& u(V, T) \\
& \left(\frac{\partial U}{\partial p}\right)_{s}=\left(\frac{\partial U}{\partial V / s}\right)\left(\frac{\partial V}{\partial p / s}\right) \\
& S \text { (constmt) }-(V-N 6) T^{3 / 2} \text { (constent) }=C \\
& T^{3 / 2}=C / V-N 6 \\
& \underline{T=(C / V-N 6)^{2 / 3}} \\
& \left.P=\frac{N k(C)^{2 / 3}}{(V-N b)^{5 / 3}}-\frac{a N^{2}}{V^{2}}=-\frac{-5}{3} \frac{N k C^{2 / 3}}{(V-N b)^{1 / 3}}+\frac{2 a N^{2}}{V^{3}}\right]=\left(\frac{, P}{\partial V}\right)_{5}=\alpha \quad \text { [cf. Eq. (18)] } \\
& U=\frac{3}{2} N k\left(\frac{C}{V-N b}\right)^{2 / 3}-\frac{a N^{2}}{V}=\left[-\left(\frac{-2}{3}\right) \frac{3}{2} N k C^{2 / 3}\left(\frac{1}{V-N b}\right)^{5 / 3}+\frac{a V^{2}}{V^{2}}=\left(\frac{\partial U}{\partial V}\right)_{s}=B \quad[\text { cf. Eq. (19)] }\right. \\
& \left(\frac{\partial U}{\partial P}\right)_{s}=\left(\frac{\partial U}{\partial V}\right)_{s}\left(\frac{\partial V}{\partial P}\right)_{s}=\frac{B}{\alpha} \\
& \text { [cf. Eq. (17) }
\end{aligned}
$$

FIG. 2. Leo's written work that demonstrates the use of constant entropy as a constraint (Sec. VA).

stating that "the other one would be the ...T term." The trailing " $+\ldots$. "seems to indicate that he was attempting to write the two-dimensional chain rule,

$$
\left(\frac{\partial U}{\partial p}\right)_{S}=\left(\frac{\partial U}{\partial V}\right)_{T}\left(\frac{\partial V}{\partial p}\right)_{S}+\left(\frac{\partial U}{\partial T}\right)_{V}\left(\frac{\partial T}{\partial p}\right)_{S}
$$

and was conflating it with a one-dimensional chain rule

$$
\left(\frac{\partial U}{\partial p}\right)_{S}=\left(\frac{\partial U}{\partial V}\right)_{S}\left(\frac{\partial V}{\partial p}\right)_{S}
$$

At this point, he did not seem to realize that the correct temperature dependence is built into the constant entropy in the one-dimensional chain rule.

Instead of pursuing the $T$ term, he focused instead on the issue of constant entropy, asking himself aloud, "How do [I] keep entropy constant?" His answer to this question was to set Eq. (2) explicitly equal to a constant:

$O k$, so this [Eq. (2)] tells me, if I want to hold the entropy constant, then $S$,

$$
S(\text { constant }) \rightarrow
$$

$S$ constant implies, [points at various parts of Eq. (2)] constants, constants, constants. Ok, so, this guy [points to numerator of logarithm in Eq. (2)] is constant.

$$
(V-N b) T^{3 / 2}(\text { constant })
$$

$O k$, so, if this is constant, then I can say, so this equals, capital C isn't taken, so

$$
=C
$$

So this tells me that

$$
T^{3 / 2}=\frac{C}{V-N b} .
$$

As long as this is true, then I feel like I'm confident that things are constant entropy ....

Leo then went on to outline his plan to solve for $U$ and $p$ as functions of only one variable, $V$ :

...So what I want to do is basically pick to solve this [points to Eq. (17)] for either $T$ or $V$ and then I want to put that in here [points to U equation of state, Eq. (3)] so that $U$ and $p$ [points to $p$ equation of state, Eq. (1)] both become a constant [sic]. So I can basically get rid of the $T$ dependence and calculate. So, now $p$ is function of $V$, and what that is, is $p$ as a function of $V$ for a constant entropy process. And then I can do the same with $U, U$ is a function of, let's say $V$, for a constant entropy process... .

After carrying out this plan and calculating both $p(V)$ and $U(V)$,

$$
\begin{gathered}
p=\frac{N k(C)^{2 / 3}}{(V-N b)^{5 / 3}}-\frac{a N^{2}}{V^{2}}, \\
U=\frac{3}{2} N k\left(\frac{C}{V-N b}\right)^{2 / 3}-\frac{a N^{2}}{V},
\end{gathered}
$$

Leo evaluated the derivative of each with respect to $V$ and returned to the one-dimensional chain rule [Eq. (15)] to calculate a final answer to the problem (see Fig. 2).

His discussion goes on to show that he recognized that by explicitly holding the entropy constant, he had reduced the problem to a one-dimensional system and had therefore resolved his earlier concern about a " $T$ term" that had resulted from his conflation of the one-dimensional and two-dimensional chain rules. However, his concern did cause him to question the "legality" of his math throughout his interview (discussed further in Sec. VIC). It is interesting to note that Leo was not the only expert who chose to set aside a difficulty while attending to other issues and return to it later when he had a new and more productive perspective.

In all cases, the primary indicator of variation 1 is a move that explicitly sets the entropy equal to a constant (or the change in entropy equal to zero). Regardless of which game one is playing, this variation reduces the degrees of freedom in the problem and is coupled with a variant in the target epistemic form.

TABLE VII. Thermodynamic potentials.

\begin{tabular}{lc}
\hline \hline Name & Fundamental equation \\
\hline Internal energy & $d U=T d S-p d V$ \\
Enthalpy & $d H=T d S+V d p$ \\
Helmholtz free energy & $d F=-S d T-p d V$ \\
Gibbs free energy & $d G=-S d T+V d p$ \\
\hline \hline
\end{tabular}




\section{B. Thermodynamic potentials}

The second variation involves using a differential of a thermodynamic potential, either the thermodynamic identity,

$$
d U=T d S-p d V
$$

or one of its Legendre transforms (see Table VII).

In the Differentials game, using a thermodynamic potential changes the set of linear equations one is trying to solve. For example, D. R. used the thermodynamic identity [Eq. (20)] instead of the differential form of the equation of state [Eq. (8)]. Sam used this variation when he equated the thermodynamic identity with the target form for the Differentials game [Eq. (5)]:

$$
d U=T d S-p d V=A d p+B d S,
$$

using placeholder variables where

$$
A=\left(\frac{\partial U}{\partial p}\right)_{S}, \quad B=\left(\frac{\partial U}{\partial S}\right)_{p} .
$$

Substituting the differential versions of the pressure and entropy equations of state [Eqs. (6) and (7)] into Eq. (21), he separately equated the resulting $d T$ and $d V$ components:

$$
\begin{gathered}
A\left(\frac{\partial p}{\partial T}\right)_{V}+B\left(\frac{\partial S}{\partial T}\right)_{V}=T\left(\frac{\partial S}{\partial T}\right)_{V} \\
A\left(\frac{\partial p}{\partial V}\right)_{T}+B\left(\frac{\partial S}{\partial V}\right)_{T}=T\left(\frac{\partial S}{\partial V}\right)_{T}-p .
\end{gathered}
$$

He then solved this set of equations for $A$, which was the desired derivative.

In the Partial Derivatives game, using the thermodynamic identity changes the goal of the problem. From the thermodynamic identity, one can see that

$$
\left(\frac{\partial U}{\partial V}\right)_{S}=-p
$$

When this is used with the one-dimensional chain rule,

$$
\left(\frac{\partial U}{\partial p}\right)_{S}=\left(\frac{\partial U}{\partial V}\right)_{S}\left(\frac{\partial V}{\partial p}\right)_{S}
$$

one essentially replaces the original goal, finding $(\partial U / \partial p)_{S}$, with the goal of finding $(\partial V / \partial p)_{S}$ :

$$
\left(\frac{\partial U}{\partial p}\right)_{S}=-p\left(\frac{\partial V}{\partial p}\right)_{S}
$$

Jay explicitly identified $(\partial V / \partial p)_{S}$ as the adiabatic compressibility and all of his moves after this one referred to finding the adiabatic compressibility as the goal [38].

One can also get to Eq. (27) by using variation 1 to eliminate the $d S$ term in the thermodynamic identity and then dividing by $d p$. Elliott and Jay did this explicitly, but Keith did so more implicitly. While considering the thermodynamic identity, he said,
TABLE VIII. Maxwell relations.

$$
\frac{\partial}{\partial x_{j}}\left(\frac{\partial \Phi}{\partial x_{i}}\right)=\frac{\partial}{\partial x_{i}}\left(\frac{\partial \Phi}{\partial x_{j}}\right)
$$

Common Maxwell relations

$$
\begin{aligned}
& \frac{\partial^{2} U}{\partial S \partial V}=+\left(\frac{\partial T}{\partial V}\right)_{S}=-\left(\frac{\partial p}{\partial S}\right)_{V} \\
& \frac{\partial^{2} H}{\partial S \partial p}=+\left(\frac{\partial T}{\partial p}\right)_{S}=+\left(\frac{\partial V}{\partial S}\right)_{p} \\
& \frac{\partial^{2} A}{\partial T \partial V}=+\left(\frac{\partial S}{\partial V}\right)_{T}=+\left(\frac{\partial p}{\partial T}\right)_{V} \\
& \frac{\partial^{2} G}{\partial T \partial p}=-\left(\frac{\partial S}{\partial p}\right)_{T}=+\left(\frac{\partial V}{\partial T}\right)_{p}
\end{aligned}
$$

So, if I look at $\partial U / \partial p$ at $S, I$ would have again $\partial S / \partial p$ at $S$, is zero and I would have, minus $p$ times partial of $V$ with respect to $p$ at $S$ [writes Eq. (27)].

This technique of dividing by a differential will be discussed further in Sec. VIC.

In addition to potentially changing the goal, using a thermodynamic potential within the Partial Derivatives game opens up additional moves through the use of Maxwell relations (Table VIII). Several experts (e.g., Elliott, Sam, D. R.) mentioned or proposed using Maxwell relations, although Keith was the only one to actually use them in his final solution.

Gary spent the majority of his time considering the various thermodynamic potentials (Table VII) and weighing the advantages and disadvantages of using each. He was one of the few experts that did not actually get to a solution.

Employing a thermodynamic potential brings in physical information in addition to the mathematical relationships between differentials and partial derivatives or the partial derivative rules. Thus, this variation implies a broader knowledge base than was strictly necessary to solve the problem mathematically. It also grounds the problem more firmly in a physical context than any of the previously discussed moves by connecting particular partial derivatives to specific thermodynamic variables.

\section{DISCUSSION}

In this section, we explore more fully some of the implications of this task analysis. We discuss some of the commonalities amongst the games presented in this paper (Sec. VI A) and consider how the games presented here compare with other previously identified epistemic games (Sec. VIB). We then highlight expert concerns about the "legality" of the mathematics in the Partial Derivatives and the Differentials games and relate this to differences in the use of partial derivatives and differentials between mathematicians and physicists (Sec. VIC). We discuss 
how differences in epistemic form imply a different conceptualization of partial derivatives (Sec. VID). Finally, we present some reflections from these experts on the nature of thermodynamics and how these reflections connect to some of the criteria they used to evaluate their progress and solutions (Sec. VIE).

\section{A. Commonalities amongst these games}

It is clear that there are several points of commonality amongst the epistemic games and variations discussed in this paper. As mentioned in Sec. II B, each of these games involves an attempt to answer the following question: Which quantities depend on which other quantities (i.e., which quantities are constant, which are independent variables, which are dependent variables, etc.)? As such, each can be considered what Collins and Ferguson [27] call functional analysis games.

In a similar way, each of these games seem to share an entry condition: to notice a mismatch between the functional relationships provided and the one required to solve the problem, e.g., the given equations of state are in terms of one set of variables $(V$ and $T)$ and the problem asks for a partial derivative with respect to different variables ( $p$ and $S)$. Although the recognition of this mismatch appears to trigger each of these games, it is unclear from these interviews why each expert chose to initially engage in one game or variation over another. We suspect that previous experience and beliefs about thermodynamics may play a significant role in when experts feel it is appropriate to play a particular game. However, we would have to delve more deeply with a different research design to uncover these connections.

There is also significant overlap in the requisite knowledge base, i.e., the cognitive and mathematical resources that are necessary to play the games. Perhaps most importantly, one must be able to recognize the multivariable nature of the problem. Additionally, the Substitution game is a necessary subgame in both of the other games. Finally, although it may be implemented at different points in each game, one must be able to actually evaluate a partial derivative.

Despite these commonalities, the games presented here diverge in interesting ways, such as in their target epistemic form and in the primary moves in each game (see Table III). These differences have important implications for instruction, some of which will be discussed in the following sections.

\section{B. Comparison to other epistemic games}

We have already discussed how the games presented in this paper are functional analysis games, as defined by Collins and Ferguson. The games identified by Lunk [29] are not applicable to the task in this paper since they are specific to computational modeling. The answer-making epistemic game that Chen et al. [28] describe also does not appear to be characteristic of these experts in this task. However, there are connections here to three of the six games that Tuminaro and Redish [34] claim are characteristic of introductory physics students (see Table IX).

One could argue that the games in this paper are all simply instances of what Tuminaro and Redish [34] call the recursive plug-and-chug game, but according to them,

... students playing this game rely only on their syntactic understanding of physics symbols, without attempting to understand these symbols conceptually.

Although a couple of these experts did not explicitly connect mathematical quantities to the physical situation, all of them did connect these quantities to the mathematical structure of the problem and to their conceptual understanding of the mathematics, which was fairly sophisticated and nuanced in some cases. In fact, the constraints that these experts placed on their mathematical moves, particularly in the Partial Derivatives game, clearly distinguished their activities from the recursive plug-and-chug game, which involves little to no evaluation of one's moves. In this sense, their actions were more akin to the mapping meaning to mathematics game or the mapping mathematics to meaning game, where the story they were telling was a mathematical story and not necessarily a physical one.

Both the mapping meaning to mathematics game and the mapping mathematics to meaning game represent high-level games that involve the interaction between the physical situation and the mathematics used to understand it. Some of the experts in this study seemed to be playing one of these games, with the Partial Derivatives game or the Differentials game played as a subgame within the higher-level game.

As mentioned in Sec. II B, partial derivatives in thermodynamics add an important nuance to the controllingvariables game [27], which is introduced as early as elementary school [39]. When students are introduced to partial derivatives in a multivariable calculus class, they are defined in the language of the controlling-variables game:

For functions of three or more variables, we find partial derivatives by the same method. Differentiate with respect to one variable, regarding the other variables as constant [40].

This is typically interpreted to mean holding everything else constant, but that is not always possible. Thus, a more nuanced interpretation is to hold constant as many variables as possible.

In most disciplines, including mathematics, the number of variables is typically known, as well as which are dependent and independent. Thus, one can use a less nuanced interpretation. In thermodynamics, these functional relationships are not always readily apparent. Therefore, one must have a more nuanced perspective, 
TABLE IX. List of epistemic games identified in Tuminaro and Redish [34].

\begin{tabular}{|c|c|}
\hline Name of the game & Description of the game \\
\hline $\begin{array}{l}\text { Mapping meaning } \\
\text { to mathematics }\end{array}$ & $\begin{array}{l}\text { "In this game, students begin from a conceptual understanding of the physical situation } \\
\text { described in the problem statement, and then progress to a quantitative solution." }\end{array}$ \\
\hline $\begin{array}{l}\text { Mapping mathematics } \\
\text { to meaning }\end{array}$ & $\begin{array}{l}\text { "In this game, students develop a conceptual story corresponding to a particular physics } \\
\text { equation." }\end{array}$ \\
\hline Physical mechanism game & $\begin{array}{l}\text { "In the Physical Mechanism Game students attempt to construct a physically coherent and } \\
\text { descriptive story based on their intuitive sense of physical mechanism." }\end{array}$ \\
\hline Pictorial analysis & $\begin{array}{l}\text { "In the Pictorial Analysis Game, students generate an external spatial representation that } \\
\text { specifies the relationship between influences in a problem statement." }\end{array}$ \\
\hline Recursive plug-and-chug & $\begin{array}{l}\text { "In the Recursive Plug-and-Chug Game students plug quantities into physics equations } \\
\text { and churn out numeric answers, without conceptually understanding the physical } \\
\text { implications of their calculations." }\end{array}$ \\
\hline Transliteration to mathematics & $\begin{array}{l}\text { "Transliteration to Mathematics is an epistemic game in which students use worked } \\
\text { examples to generate a solution without developing a conceptual understanding of } \\
\text { the worked example." }\end{array}$ \\
\hline
\end{tabular}

and functional analysis games like those described here are vital to understanding the situation. In fact, some of the errors made by experts in this study appeared to be due to a failure to attend to this nuance.

\section{C. "Mathematically illegal"}

The disconnect between mathematicians and physicists was evident in several experts who expressed the concern that one or more of their moves might not be considered acceptable to a mathematician. We discuss two such examples here.

In the Partial Derivatives game, the primary moves are the partial derivative rules in Table IV, which one must be able to remember or reproduce accurately. As discussed in Sec. VA, Leo conflated the one-dimensional [Eq. (15)] and two-dimensional chain rules [Eq. (14)] when he started the problem. Although he ultimately resolved the issue by explicitly holding the entropy constant, he questioned whether his math was "legal" throughout the interview.

So, I feel like what I'm writing [Eq. (15)] is not quite mathematically, sort of, legal, but I think it's, but I think it's physically legal.

When asked to explain further, he said

The legal illegal part is why I don't have a $\partial U / \partial T$ term in here [Eq. (15)] and I think that, I feel like that's ok because I'm thinking about a process, so I'm really thinking about small changes in each of these [points to $U$ and $p$ in the $(\partial U / \partial p)_{S}$ term] as entropy [points to S] stays constant. So, I think that's fine because these are both [points to $p(V)$ and $U(V)$, Eqs. (18) and (19)], the temperature changes are built into these, both of these pieces.

He correctly recognized that he had "built in" the temperature dependence by explicitly holding the entropy constant, but was still unsure of how a mathematician would view his work.

Another point of contention between mathematicians and physicists is the use of differentials. A common practice among the experts in this study was to divide by a differential, which Elliott referred to as "that bogus physicist-y way" of turning a differential into derivative. When considering the thermodynamic identity [Eq. (20)], he set $d S=0$ and then stated,

So now I have $d U$ is minus pdV and then I thought about trying to make that into a partial derivative in sort of that bogus physicist-y way by treating it as a fraction and then just ignoring the fact that it's, I mean just sort of changing that fraction into a derivative in some sort of sneaky way. So on the left-hand side I have partial $\partial U / \partial p$ with $S$ held constant and then I have to sort of take the other side, over dp basically.

$$
\left(\frac{\partial U}{\partial p}\right)_{S}=-\left[\frac{\partial}{\partial p}(p d V)\right] .
$$

And I have to be a little careful here, I think because, I have to use, do I have to use some sort of product rule thing here? Huh.

Elliott chose to set his issue aside, and when he returned to it, he resolved the issue by looking at the one-dimensional chain rule [Eq. (15)] and explicitly considering whether he had held entropy constant.

Unlike most mathematicians, physicists are willing to work with differentials as intuitive objects standing for small changes [21]. Yet, as Elliott demonstrated, physicists are not always sure of the legitimacy of their use of differentials from a mathematics perspective.

Given the disconnect between mathematics and physics use as discussed above, physicists clearly do not learn these views in their math courses, implying that they are passed on 
in a physics context instead. It should therefore not be surprising that the language and notation that physicists have developed to deal with partial derivatives in thermodynamics may seem to be at odds with a pure mathematics approach. Nor should we be surprised that students struggle to make sense of the complex functional structures within thermodynamics, given the nuance required. Two of the authors (T.D. and C. A. M.) are working on a paper that more fully explores how the disconnect between mathematicians and physicists impacts the use of differentials in the classroom.

\section{Concept of partial derivatives}

One of the most notable differences between the games presented in this paper is in the target epistemic form (see Table III). For example, the goal of the Substitution game (when played by itself) is to solve for and differentiate an explicit expression for internal energy as a function of pressure and entropy. Yet both the Partial Derivatives game and the Differentials game bypass this goal through differentiation.

More importantly, the difference in epistemic form between the Partial Derivatives game and the Differentials game suggests a fundamental difference in how one conceptualizes a partial derivative. For example, after being prompted to use differentials, Jay reflected that

... essentially this is a different encoding of the same information. So, if you think about it, I mean, I don't see off-hand any reason why this encoding is different from writing as partial derivatives. I mean the form is different, but I think the encoding is the same.

When the interviewer asked him to clarify, Jay went on to more clearly distinguish between these two forms.

If you think about small changes

$$
\Delta x, \Delta y, \Delta z
$$

whatever they are, you find equations that relate those things ... you write ... the changes as changes in themselves and not how they're related to each other 'cause that you solve [for]... later on by picking out which of these [changes] are constant. I mean the other approach, where you write things like

$$
\left(\frac{\partial x}{\partial y}\right)_{z}
$$

...here [points to Eq. (29)] you work with variables, here [points to Eq. (30)] you work with ratios of variables, directly, but you have to make sure you pick the right ratios. So, you have the same information. Here [points to Eq. (29)] you have the independent changes and ... you have the freedom to connect them somehow. Here [points to Eq. (30)] you have them as dependent changes because you say ok there's a, out of these [Eq. (29)] I can construct ... three different ratios ... and I'm not sure which one is the best.
According to Jay, in the differentials game, a partial derivative is a ratio of small independent changes, whereas in the Partial Derivatives game, it is one particular dependent change. While both contain the same information, they are "encoded" differently.

Similarly, there is a difference between a partial derivative at a point, which is a ratio of small quantities, and the idea of a derivative as an operation on functions. Experts appear to be able to move fluidly between these ideas, often not distinguishing between them. Yet, these different conceptualizations present a pedagogical challenge and, thus, are particularly relevant to thinking about what we want our students to be able to do. The theoretical framework proposed by Zandieh [17] and applied to student understanding of ordinary derivatives would be an excellent way to explore this area further.

\section{E. Nature of thermodynamics}

Several experts commented on the fact that it was possible to find a solution that was independent of the specific functional form of the equations of state. Those that did seemed to feel that this characteristic distinguished thermodynamics from other subdisciplines in physics. According to Elliott,

... the place where we get Maxwell relations and all the partial derivative stuff that you do in thermo, to me, ... it's just calculus, if you have multi-variable calculus ... and it's not specific to any system."

And Chris pointed out,

One of the challenges... and it's not a challenge, it's the power of thermodynamics, ... the formalism is independent of any underlying model ... It's a different way of thinking about it and so, you know, in that sense, it's a little bit unappealing for lots of people ... but ... classical thermo is a beautiful theory.

This view of thermodynamics as an abstract mathematical formalism is consistent with the ways that most of these experts treated this problem. For example, when evaluating their progress, they tended to focus on aspects related to the mathematical structure of the problem such as whether a given step produced a "nice set."

Despite statements like those above, when it comes to the nature of thermodynamics, these experts seem to simultaneously hold two perspectives in tension. This was evident in how they attempted to evaluate their solutions, as opposed to their progress. Instead of the mathematical structure, they focused on the physical model and physical intuition. For example, after trying one approach, Elliott decided to evaluate his expectations:

I'm gonna stop for a second and just think about what I expect to happen. Maybe I should have done that earlier. So, if I did the experiment I did in one, insulated, 
slow, no heat transfer, add mass to it, I do work on the gas, um, as I increase the pressure, the volume's going to decrease. [pause] It's a van der Waals gas, so two competing effects, one of which is that the particle's solid. If I get to a sufficiently dense gas, I have to worry about the actual volume of the particles themselves. That is what little $b$ means and then I also have to think about the fact that there's an attractive force between the particles. So, if the volume decreases, what'll I expect to happen to the internal energy? If there was no interactions [sic] between the particles, it would of course increase cause I do positive work on the gas. Well, heck, what am I thinking, so if I [do] positive work on the gas, the internal energy has to increase. That's not ambiguous, I should know that.

Similarly, both Leo and Chris attempted to evaluate their solution in terms of a limiting case (i.e., an ideal gas). However, Leo was unsatisfied by not having an intuition for what the answer should be in this case,

Oh, I'm just, I'm having trouble convincing myself that this answer [for an ideal gas], I mean it seems reasonable, but it, but I don't have a good way of saying, and I mean I guess [what] I could do is look back to my process and say, do I believe all the steps? I'm, you know, I'm slightly comforted by the fact that the units work out right, but I'd like some kind of other support.

There seem to be two aspects of thermodynamics, which these experts hold in tension and move between fluidly. On the one hand, it is an abstract mathematical formalism that allows one to derive general relationships that will hold regardless of the physical system. On the other hand, any evaluation of meaning (or correctness of a solution) is usually based more on what that formalism tells us about a specific system, such as an ideal gas or van der Waals gas. Understanding expert views about the nature of thermodynamics and how these views are held in tension has significant implications for our instruction and for helping our students to develop professionally.

\section{CONCLUSIONS}

In this paper, we have used epistemic games as an analytic tool for performing a cognitive task analysis. Employing this tool, we have conducted a task analysis on a mathematical problem in thermodynamics and identified three epistemic games and two variations that represent expert approaches to this problem. This analysis has led to several interesting insights into expert use and understanding of the mathematics of thermodynamics and to several areas for further exploration.

In this section, we discuss some of the limitations of this study, briefly summarize the major implications of this work and some current areas of research that have grown from it, as well as suggest further lines of inquiry.

\section{A. Limitations}

As with all research, there are some limitations to the conclusions that we can draw from the data in this study.

Although we attempted to recruit faculty with expertise in thermodynamics, we cannot evaluate the representativeness of our sample.

We recognize the inherent sociocultural nature of practice and that, for physics professionals, problem solving is often a collaborative exercise. This aspect of practice was not represented in these individual interviews. The use of epistemic games as an analytic tool would be equally valuable (if not more so) in a setting which focuses on the sociocultural aspects of problem solving.

We intentionally posed a problem that was inherently mathematical in nature and had clear initial and final conditions. Although more challenging for experts than introductory-level problems, it is still artificial. This is also not the only kind of problem in thermodynamics and perhaps not the most important. Given the mathematical nature of the problem, this analysis has more to say about how physicists understand and use the mathematics of thermodynamics than about how they conceptualize the physics of thermodynamics: an important distinction that must be explored.

In categorizing the behavior of these experts through the use of epistemic games, we do not claim that those discussed here are a complete set. There were moves that some experts made that we chose not to mention here, primarily because the experts themselves chose to abandon these approaches fairly early in the process. Some of these moves may be part of the games discussed here or may constitute other games altogether. In order to explore them more fully, one must provide tasks that focus on different aspects of thermodynamics.

For example, some of the experts (Leo, Elliott, Keith, and Chris) answered another prompt before the van der Waals question:

Draw and describe an experiment that would measure the quantity:

$$
\left(\frac{\partial U}{\partial p}\right)_{S}
$$

This type of question is used extensively in the Energy and Entropy course [18] at Oregon State University. "Name the experiment" activities, such as this question, are described in Roundy et al. [41]. This prompt was designed to cue a process where the primary goal is to answer the question, "How can the quantity $X$ [in this case, the derivative $(\partial U / \partial p)_{S}$ ] be physically measured?" For example, in answering this question, Elliott stated

This is an adiabatic process and so I can figure out, basically the change in internal energy is gonna be equal to the work done and so I can take integral $p d V$, stick a negative sign in front of it and that would give me the work, which would also be the change in internal energy. 
Both Leo and Elliott began the van der Waals problem by returning to the idea of finding the work done, both explicitly mentioning the "name the experiment" question. Although Elliott pursued this path to the point of attempting to evaluate an integral, both he and Leo ultimately recognized that the van der Waals problem was not particularly solvable with this approach and abandoned it in favor of the Partial Derivatives game.

The fact that the earlier problem cued an approach that was unproductive in this case is important confirmation that performance is to some extent context dependent. It would be worthwhile to consider what experts do with a problem where the kind of physical reasoning that is important in the "name the experiment" question would also be helpful.

\section{B. Implications and future work}

In our discussion in Sec. VI, we presented several significant areas of interest that emerged from a comparison of the games presented in this paper. Here we briefly revisit these areas and discuss current and future work exploring these issues further.

The Partial Derivatives game and the Differentials game represent two very different ways of conceptualizing a partial derivative. The recognition of this difference has prompted a study that more fully explores how experts in different disciplines understand partial derivatives. We are now in the process of conducting group interviews where experts in different STEM (science, technology, engineering, and mathematics) fields explore the use of partial derivatives in their field. We intend to use this research to inform curriculum development in both mathematics and upper-division physics.

The disconnect between mathematics and physics was apparent in how some of these experts discussed the "legality" of their mathematics and raised an important issue regarding where and how our students learn to play these games. As Morrison and Collins [30] point out,
... you learn how to play ... simply and only by playing these games with people who are already relatively more fluent than you are-and who, crucially, are willing to gradually pull you up to their level of expertise by letting you play with them.

The issue related to holding "everything else" constant has led to the development of a mechanical apparatus, which we call a Partial Derivative Machine, that is designed to be a mechanical analogue of a thermodynamic system. We expect this system will allow students to explore the impact of which quantities are held constant in a setting where they can use their intuition about mechanics [42].

The experts in this study clearly recognize the complexity and beauty of the mathematical formalism of thermodynamics. Yet, they have also developed the ability to focus on the conceptual and physical story when needed. This interplay between complex mathematical structure and physical understanding and intuition is particularly important to cultivate in a field like thermodynamics where students often cannot make the connections between the unfamiliar mathematical techniques and the unfamiliar physical quantities like entropy. We plan to explore how activities designed to provide more physical significance to thermodynamics through concrete examples [41-44] can help students to make connections between the mathematics and the physics.

\section{ACKNOWLEDGMENTS}

The authors wish to thank each of the faculty who participated in this study, as well as Emily van Zee for her continued work in our on-going understanding of the "Name the Experiment" activities. Finally, the authors gratefully acknowledge the support of the National Science Foundation through Grants No. DUE 0088901, No. 0837829, and No. 1023120. This work is part of the Paradigms in Physics Project at Oregon State University.
[1] David P. Maloney, in Getting Started in PER, Reviews in PER Vol. 2, edited by Charles Henderson and Kathleen Harper (American Association of Physics Teachers, College Park, MD, 2011).

[2] Chandralekha Singh, When physical intuition fails, Am. J. Phys. 70, 1103 (2002).

[3] Thomas J. Bing and Edward F. Redish, Analyzing problem solving using math in physics: Epistemological framing via warrants, Phys. Rev. ST Phys. Educ. Res. 5, 020108 (2009).

[4] Marcos D. Caballero, Bethany R. Wilcox, Rachel E. Pepper, and Steven J. Pollock, A framework on the use of mathematics in upper-division physics,
Phys. Rev. ST Phys. Educ. Res. 9, 020119 (2013).

[5] Joseph F. Wagner, Corinne A. Manogue, and John R. Thompson, Representation issues: Using mathematics in upper-division physics, AIP Conf. Proc. 1413, 89 (2012).

[6] Ana R. Ataíde and Ileana M. Greca, Epistemic views of the relationship between physics and mathematics: Its influence on the approach of undergraduate students to problem solving, Sci. Educ. 22, 1405 (2013).

[7] Trevor I. Smith, Warren M. Christensen, and John R. Thompson, Addressing student difficulties with concepts related to entropy, heat engines and the Carnot cycle, AIP Conf. Proc. 1179, 277 (2009). 
[8] Michael E. Loverude, Student understanding of basic probability concepts in an upper-division thermal physics course, AIP Conf. Proc. 1179, 189 (2009).

[9] Brandon Bucy, John Thompson, and Donald Mountcastle, Student (mis)application of partial differentiation to material properties, AIP Conf. Proc. 883, 157 (2007).

[10] Warren M. Christensen and John R. Thompson, in Proceedings of the 13th Annual Conference on Research in Undergraduate Mathematics Education (Mathematical Association of America, Oberlin, OH, 2010), http:// sigmaa.maa.org/rume/crume2010/Abstracts2010.htm.

[11] John R. Thompson, Brandon R. Bucy, and Donald B. Mountcastle, Assessing student understanding of partial derivatives in thermodynamics, AIP Conf. Proc. 818, 77 (2006).

[12] John R. Thompson, Corinne A. Manogue, David J. Roundy, and Donald B. Mountcastle, Representations of partial derivatives in thermodynamics, AIP Conf. Proc. 1413, 85 (2012).

[13] Warren M. Christensen and John R. Thompson, Investigating graphical representations of slope and derivative without a physics context, Phys. Rev. ST Phys. Educ. Res. 8, 023101 (2012).

[14] Mark Asiala, Jim Cottrill, Ed Dubinsky, and Keith E. Schwingendorf, The development of students' graphical understanding of the derivative, J. Math. Behav. 16, 399 (1997).

[15] John S. Berry and Melvin A. Nyman, Promoting students' graphical understanding of the calculus, J. Math. Behav. 22, 479 (2003).

[16] Anthony Orton, Students' understanding of differentiation, Educ. Stud. Math. 14, 235 (1983).

[17] Michelle J. Zandieh, in Research in Collegiate Mathematics Education IV, CBMS Issues in Mathematics Education, edited by E. Dubinsky, A.H. Schoenfeld, and J. Kaput (American Mathematical Society, Providence, RI, 2000), p. 103.

[18] Paradigms in Physics: Course materials, http:// physics.oregonstate.edu/portfolioswiki/courses:start.

[19] Vector Calculus Bridge Project, http://physics.oregonstate .edu/bridge.

[20] Tevian Dray and Corinne Manogue, Using differentials to bridge the vector calculus gap, Coll. Math. J. 34, 283 (2003).

[21] Tevian Dray and Corinne A. Manogue, Putting differentials back into calculus, Coll. Math. J. 41, 90 (2010).

[22] Dan Diaper, in The Handbook of Task Analysis for Human-Computer Interaction, edited by Dan Diaper and Neville Stanton (Lawrence Erlbaum Assoc., Mahwah, NJ, 2004).

[23] The Cambridge Handbook of Expertise and Expert Performance, edited by K. Anders Ericsson, Neil Charness, Paul J. Feltovich, and Robert R. Hoffman (Cambridge University Press, New York, 2006).

[24] Jan Maarten Schraagen, in The Cambridge Handbook of Expertise and Expert Performance, edited by $\mathrm{K}$. Anders Ericsson, Neil Charness, Paul J. Feltovich, and Robert R. Hoffman (Cambridge University Press, New York, 2006), Chap. 11.
[25] Cognitive Task Analysis, edited by Jan Maarten Schraagen, Susan F. Chipman, and Valerie Shalin (Lawrence Erlbaum, Mahwah, NJ, 2000).

[26] Thomas J. Bing and Edward F. Redish, Epistemic complexity and the journeyman-expert transition, Phys. Rev. ST Phys. Educ. Res. 8, 010105 (2012).

[27] Allan Collins and William Ferguson, Epistemic forms and epistemic games: Structures and strategies to guide inquiry, Educ. Psychol. 28, 25 (1993).

[28] Ying Chen, Paul W. Irving, and Eleanor C. Sayre, Epistemic game for answer making in learning about hydrostatics, Phys. Rev. ST Phys. Educ. Res. 9, 010108 (2013).

[29] Brandon R. Lunk, Ph.D. thesis, North Carolina State University, 2012.

[30] Donald Morrison and Allan Collins, Epistemic fluency and constructivist learning environments, Educ. Technol. 35, 39 (1995).

[31] Edward F. Redish and Karl A. Smith, Looking beyond content: Skill development for engineers, J. Eng. Educ. 97, 295 (2008).

[32] Lorraine Sherry and Maggie Trigg, Epistemic forms and epistemic games, Educ. Technol. 36, 38 (1996).

[33] Jonathan Tuminaro, Ph.D. thesis, University of Maryland, 2004.

[34] Jonathan Tuminaro and Edward F. Redish, Elements of a cognitive model of physics problem solving: Epistemic games, Phys. Rev. ST Phys. Educ. Res. 3, 020101 (2007).

[35] David N. Perkins, Epistemic games, Int. J. Educ. Res. 27, 49 (1997).

[36] This should not be confused with the branch of mathematics which is also called functional analysis.

[37] Sam's interview consisted of a discussion of his written solution to the problem, which he had solved prior to the interview.

[38] Mary Bridget Kustusch, David Roundy, Tevian Dray, and Corinne A. Manogue, An expert path through a thermo maze, AIP Conf. Proc. 1513, 234 (2013).

[39] Taking Science to School: Learning and Teaching Science in Grades $K-8$, edited by Richard A. Duschl, Heidi A. Schweingruber, and Andrew W. Shouse (National Academies Press, Washington, DC, 2007).

[40] William G. McCallum, Deborah Hughes-Hallett, and Andrew M. Gleason, Multivariable Calculus (John Wiley \& Sons, New York, 2005), 4th ed.

[41] David Roundy, Mary Bridget Kustusch, and Corinne A. Manogue, Name the experiment!, Am. J. Phys. 82, 39 (2014).

[42] Grant Sherer, Mary Bridget Kustusch, Corinne A. Manogue, and David Roundy, "The partial derivative machine," AIP Conf. Proc. (to be published).

[43] Corinne A. Manogue, David Roundy, Mary Bridget Kustusch, Emily H. van Zee, Tevian Dray, Ayush Gupta, and Joseph Wagner, "From fear to fun in thermodynamics: Multiple research perspectives for assessing learning during a curricular sequence," AIP Conf. Proc. (to be published).

[44] David Roundy and Michael Rogers, Exploring the thermodynamics of a rubber band, Am. J. Phys. 81, 20 (2013). 